DOI: 10.15586/jomh.v16i3.246

\title{
FEBRILE URINARY TRACT INFECTION AFTER RADICAL CYSTECTOMY WITH URINARY DIVERSION: DIFFERENT CHARACTERISTICS IN PATIENTS WITH ILEAL CONDUIT AND ORTHOTOPIC NEOBLADDER
}

\author{
Seungsoo Lee ${ }^{1}$, Seung Ryong Baek${ }^{1}$, Won Hoon Song ${ }^{1}$, Tae Nam Kim², Sung-Woo Park', Jong Kil Nam¹ \\ ${ }^{1}$ Department of Urology, Pusan National University Yangsan Hospital, Yangsan, Korea \\ ${ }^{2}$ Department of Urology, Pusan National University Hospital, Busan, Korea \\ Corresponding author: Jong Kil Nam: tuff-kil@hanmail.net
}

Submitted: 28 March 2020. Accepted: 05 June 2020. Published: 27 August 2020.

\begin{abstract}
Background and Objective

To compare the incidence of febrile urinary tract infection (UTI) and bacterial identification between patients with orthotopic neobladder (ONB) and ileal conduit (IC).

\section{Materials and Methods}

Data of 164 patients who underwent radical cystectomy with ONB and IC for bladder cancer between January 2009 and January 2018 at our institution were analyzed. Febrile UTI observed was listed and subsequently compared. Incidence of febrile UTI, clinicopathological characteristics, and microorganisms identified were reported at 3 months interval; and preoperative predictors of febrile UTI were evaluated with Cox regression analysis. Patients were divided into ONB and IC.
\end{abstract}

\section{Results}

The study cohort included 52 patients with ONB and 112 patients with IC. Febrile UTI was diagnosed in 49 $(29.9 \%)$ patients. Compared to IC group, ONB group had significantly higher incidence of young age $(\mathrm{p}=0.00)$, lower cancer stage $(\mathrm{p}=0.013)$, longer hospital stay $(\mathrm{p}=0.049)$, longer operation time $(\mathrm{p}=0.00)$, and higher incidence of febrile UTI within the first 3 months after surgery $(\mathrm{p}=0.006)$. On univariable and multivariable analysis, factors associated with significantly increased febrile UTI risk were diabetes (odds ratio [OR]: 4.52; $\mathrm{p}=0.002$ ) and ONB diversion (OR: $1.27 ; \mathrm{p}=0.031)$. Forty-four $(89.8 \%)$ patients were culture positive. However, significant difference in microorganisms was not detected between patients who under-went ONB or IC diversion.

\section{Conclusion}

Diabetes and ONB diversion were associated with higher risk symptomatic UTI following radical cystectomy. 
Keywords: urinary tract infections; urinary bladder neoplasms; urinary diversion

\section{INTRODUCTION}

Radical cystectomy with pelvic lymph node dissection followed by the formation of urinary diversion represents a standard of care for muscle invasive bladder cancer., ${ }^{1,2}$ The most common diversion types are orthotopic neobladder (ONB) and ileal conduit (IC). ${ }^{3}$ However, urinary diversion is associated with many complications, including bowel obstruction, prolonged ileus, urinary tract infections (UTIs), stoma problem, ureteral stricture, metabolic acidosis, electrolyte imbalance, urolithiasis, and renal dysfunction. ${ }^{4}$ Improvements in surgical technique and modern perioperative care have diminished perioperative complications rate substantially.

However, febrile UTI occurs frequently in patients who have undergone radical cystectomy. ${ }^{5-7}$ In most cases, managements are complicated due to accompanying conditions, thus affecting prognosis of patients.

The objective of this study was to analyze the incidence, timing, risk factors, and microorganisms of febrile UTI in patients with bladder cancer treated by radical cystectomy according to methods of urinary diversion after radical cystectomy.

\section{MATERIALS AND METHODS}

This study was approved by the Institutional Review Board of our hospital (Approval No. 06-2014-006). Between January 2009 and January 2018 , a total of 184 patients at our hospital underwent radical cystectomy with urinary diversion by a single surgeon (JKN), including 164 patients who had been observed at least 2 years postoperatively.
We excluded patients who didn't undergo a postoperative follow-up of less than 2 years $(n=10)$, patients who died within 2 years $(n=6)$, and patients who had undergone another major surgery $(n=4)$.

These 164 patients were used as subjects of this study. Indications for radical cystectomy included muscle invasive bladder cancer without distant metastasis, recurrent multifocal super cial tumor refractory to repeated transurethral resection, and bacillus Calmette-Guerin resistant carcinoma in situ. Radical cystectomy with urinary diversion was performed using standard technique. ONB was performed as Studer's ONB technique. Patients underwent urinary catheterization with Foley catheter and neobladder irrigation for 2 weeks in general. In the IC surgery, ureters were spatulated and anastomosed independently to the ileal segment. In both surgeries, ureteral stents were inserted for 8 weeks in general.

Preoperative urine culture was obtained from all patients, and if positive, we underwent radical cystectomy with urinary diversion after treatment. In all patients, low residual diet was performed from 2 days before surgery and bowel preparation was performed in the night before surgery. Follow-up was conducted every 3 months during the rst postoperative year and every 6 months thereafter. We performed computed tomography or magnetic resonance imaging to recurrence, laboratory study including urine culture, and a medical examination by interview. Febrile UTI was d ned as a pyuria in the presence of fever $\left(\geq 38^{\circ} \mathrm{C}\right)$, chills with or without associate ank or abdominal pain.

Febrile UTI observed was prospectively listed and subsequently compared. Baseline 
clinicopathological characteristics abstracted included age, sex, weight, height, smoking status, diabetes, stage, grade, Clavien-Dindo classi cation of surgical complications, length of hospital stays, operative time, estimated blood loss, chemotherapy, and timing of febrile UTI.

Those who were aged $\geq 65$ years were considered as elderly patients. Body mass index was dichotomized as obese $\left(\geq 25 \mathrm{~kg} / \mathrm{m}^{2}\right)$ versus non-obese $(<25$ $\mathrm{kg} / \mathrm{m}^{2}$ ) according to WHO proposed cut points for adult Asians. ${ }^{8}$ Pathological stage was based on 2010 American Joint Committee on cancer TNM staging system for bladder cancer. ${ }^{9}$ Urinary diversion was classi ed as two groups: ONB and IC. Incidence of febrile UTI, clinicopathological characteristics, and growth of microorganism were compared between the two diversion method groups. Mann-Whitney $\mathrm{U}$ test and Fisher's exact test were used to compare categorical variables.

Estimated probability of febrile UTI was calculated with Kaplan-Meier method, and predictors of febrile UTI were evaluated using univariable and multivariable Cox regression models. Any $p$-value of less than 0.05 was considered statistically signi cant. Statistical analyses were performed using SPSS software version 18.0.

\section{RESULTS}

A total of 164 patients were eligible for this comparative analysis. The mean age of the patients was 68 years (range, 40-84) with average follow-up in patients who are alive at 62.8 months (range, 24-132). Of these individuals, 52 received ONB diversion, and 112 received IC diversion. A total of $49(29.9 \%)$ patients were diagnosed with febrile UTI. Clinicopathological characteristics of these subjects are summarized in Table 1 .

All the patients with Clavien-Dindo classi cation 3 complication underwent insertion of percutaneous nephrostomy or ureteral stent for UTI. In some cases, temporary anastomosis site stricture caused UTI. In addition, the patients with Clavien-Dindo classi cation 4 complication underwent intensive care unit care due to severe UTI. All those cases were recovered from the complications, and there were no recurrence UTI in 3 months.

Compared to IC group, ONB group had signi cantly higher incidence of young age $(p<0.001)$, dominant male patients $(p=0.019)$, lower cancer stage $(p=0.013)$, longer hospital stay $(p=0.049)$, and longer operation time $(p<0.001)$. During the rst 3 months after surgery, $26.9 \%$ of the patients with ONB and $8 \%$ of the patients with IC had a febrile UTI episodes $(p=0.006)$. Figure 1 compares the total percentage of infections at 3 months interval during the rst 2 years after surgery for patients with ONB and IC diversion.

We investigated on the basis of rst febrile UTI within 3 months. Table 2 reports univariable and multivariable analyses of the predictors of febrile UTI. On univariable analysis, factors associated with signi cantly increased febrile UTI risk were current smoker $(p=0.041)$, diabetes $(p<0.001)$, and ONB diversion $(p=0.014)$. On multivariable analysis, factors associated with febrile UTI risk were diabetes (odds ratio [OR]: 4.52; $95 \%$ con dence interval [CI]: 2.862-5.571; $p=0.002$ ) and ONB diversion (OR: 2.563; 95\% CI: $1.630-2.948 ; p=0.031$ ) (Table 2). Forty-four (89.8\%) patients were culture positive. However, signi cant difference in microorganisms was not detected between patients who underwent ONB or IC diversion (Table 3). Table 3 details the common pathogens involved in febrile UTI episodes stratied by diversion type.

Escherichia coli (20\%), Enterococcus faecium (20\%), and Pseudomonas aeruginosa (16\%) were common causative pathogens in patients with ONB; patients with IC had comparable rates of Enterococcus faecium (16\%), E. coli (16\%), Klebsiella pneumoniae (16\%), Candida albicans (11\%), and Staphylococcus aureus (11\%). However, signi cant differences in microorganisms were not detected among the two groups of patients according to urinary diversion. 
TABLE 1 Clinicopathological Characteristics of Patients

\begin{tabular}{|c|c|c|c|c|c|}
\hline \multirow{2}{*}{\multicolumn{2}{|c|}{ Mean age }} & Total cohort $(n=164)$ & ONB $(n=52)$ & IC $(n=112)$ & $p$-value \\
\hline & & $68(40,84)$ & $62.6(40,77)$ & $70.0(40,84)$ & $<0.001$ \\
\hline \multicolumn{2}{|c|}{ Sex (Male:Female) } & 140: 24 & $50: 2$ & $90: 22$ & 0.019 \\
\hline \multicolumn{2}{|l|}{ Mean BMI } & 22.78 & 23.66 & 22.66 & 0.189 \\
\hline \multirow[t]{2}{*}{ Current smoker } & Yes & 41 & 10 & 31 & 0.121 \\
\hline & No & 123 & 42 & 81 & \\
\hline \multirow[t]{2}{*}{ Diabetes } & Yes & 34 & 8 & 26 & 0.132 \\
\hline & No & 130 & 44 & 86 & \\
\hline \multirow[t]{5}{*}{ Stage } & $\leq \mathrm{pT} 1$ & 27 & 14 & 13 & 0.013 \\
\hline & pT2 & 39 & 10 & 29 & \\
\hline & pT3 & 68 & 24 & 44 & \\
\hline & pT4 & 30 & 4 & 26 & \\
\hline & $\mathrm{pN}+$ & 42 & 11 & 31 & 0.468 \\
\hline \multirow[t]{3}{*}{ Grade } & 1 & 1 & 0 & 1 & 0.646 \\
\hline & 2 & 59 & 17 & 42 & \\
\hline & 3 & 104 & 35 & 69 & \\
\hline \multicolumn{2}{|c|}{$\begin{array}{l}\geq 2 \text { Complication (within } 3 \text { months, } \\
\text { Clavien-Dindo classi cation) }\end{array}$} & 69 & 30 & 39 & 0.736 \\
\hline & 2 & 51 & 23 & 28 & \\
\hline & 3 & 14 & 6 & 8 & \\
\hline & 4 & 4 & 1 & 3 & \\
\hline \multicolumn{2}{|c|}{ Febrile UTI (within 3 months) } & $24(14.6 \%)$ & $14(26.9 \%)$ & $10(8.9 \%)$ & 0.006 \\
\hline \multicolumn{2}{|c|}{ Mean length of admission (day) } & $17(9,55)$ & $20.6(10,38)$ & $18.4(9,41)$ & 0.049 \\
\hline \multicolumn{2}{|c|}{ Mean operative time (hour) } & $4(2,11)$ & $6.17(3,11)$ & $4.76(2,10)$ & $<0.001$ \\
\hline \multicolumn{2}{|c|}{ Median estimated blood loss (L) } & $1.00(0.1,3.5)$ & $1.00(0.1,3)$ & $0.99(0.1,3.5)$ & 0.722 \\
\hline
\end{tabular}

ONB, orthotopic neobladder; IC, ileal conduit; BMI, body mass index; UTI, urinary tract infection.

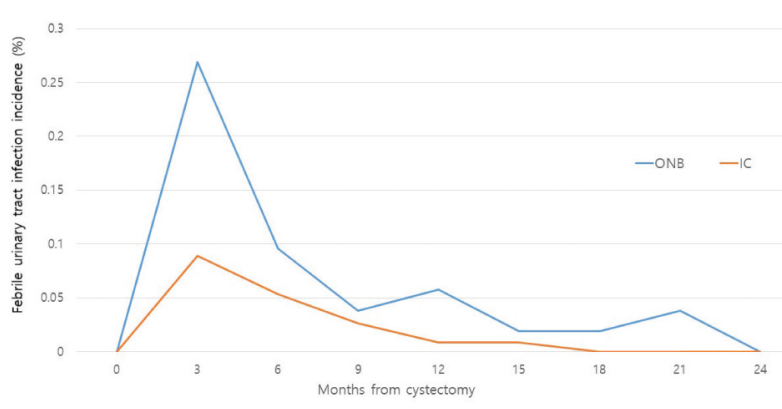

FIG. 1 Rates of febrile urinary tract infection within the rst 3 months after surgery according to diversion type.

\section{DISCUSSION}

Febrile UTIs after radical cystectomy include ank pain, nausea, vomiting, high fever, with or without costovertebral angle tenderness..$^{10}$ It is a common infectious complication following urinary diversion. In large series of patients undergoing radical cystectomy with urinary diversion, the rate of UTI after radical cystectomy ranges from 8.5 to $39 \% .^{5-7,11-14}$ Its cumulative incidence during the rst 3 months after surgery is about $10 \%{ }^{15}$ However, diagnostic criteria for UTI utilized in these series 
TABLE 2 Univariate and Multivariate Analysis of Factors Associated with Febrile Urinary Tract Infection (within 3 Months)

\begin{tabular}{|l|c|c|c|c|c|c|}
\hline & \multicolumn{3}{|c|}{ Univariate analysis } & \multicolumn{3}{c|}{ Multivariate analysis } \\
\cline { 2 - 7 } & Odds ratio & $\mathbf{9 5 \%}$ CI & $\boldsymbol{p}$-value & Odds ratio & $\mathbf{9 5 \%}$ CI & $\boldsymbol{p}$-value \\
\hline Age $(<65$ years) & 0.758 & $0.382-1.504$ & 0.267 & - & - & - \\
\hline Sex (male) & 0.383 & $0.124-1.185$ & 0.066 & - & - & - \\
\hline Current smoker (yes) & 1.892 & $0.922-3.883$ & 0.041 & - & - & - \\
\hline Diabetes (yes) & 5.000 & $3.584-6.944$ & $<0.001$ & 4.521 & $2.862-5.571$ & 0.002 \\
\hline Obesity (BMI $\geq 25)$ & 0.887 & $0.400-1.969$ & 0.468 & - & - & - \\
\hline pT stage (3/4) & 1.256 & $0.638-2.474$ & 0.314 & - & - & - \\
\hline ONB & 2.563 & $1.781-3.125$ & 0.014 & 2.273 & $1.630-2.948$ & 0.031 \\
\hline
\end{tabular}

CI, confidence interval; BMI, body mass index; ONB, orthotopic neobladder.

TABLE 3 Cultured Microorganisms according to Urinary Diversion

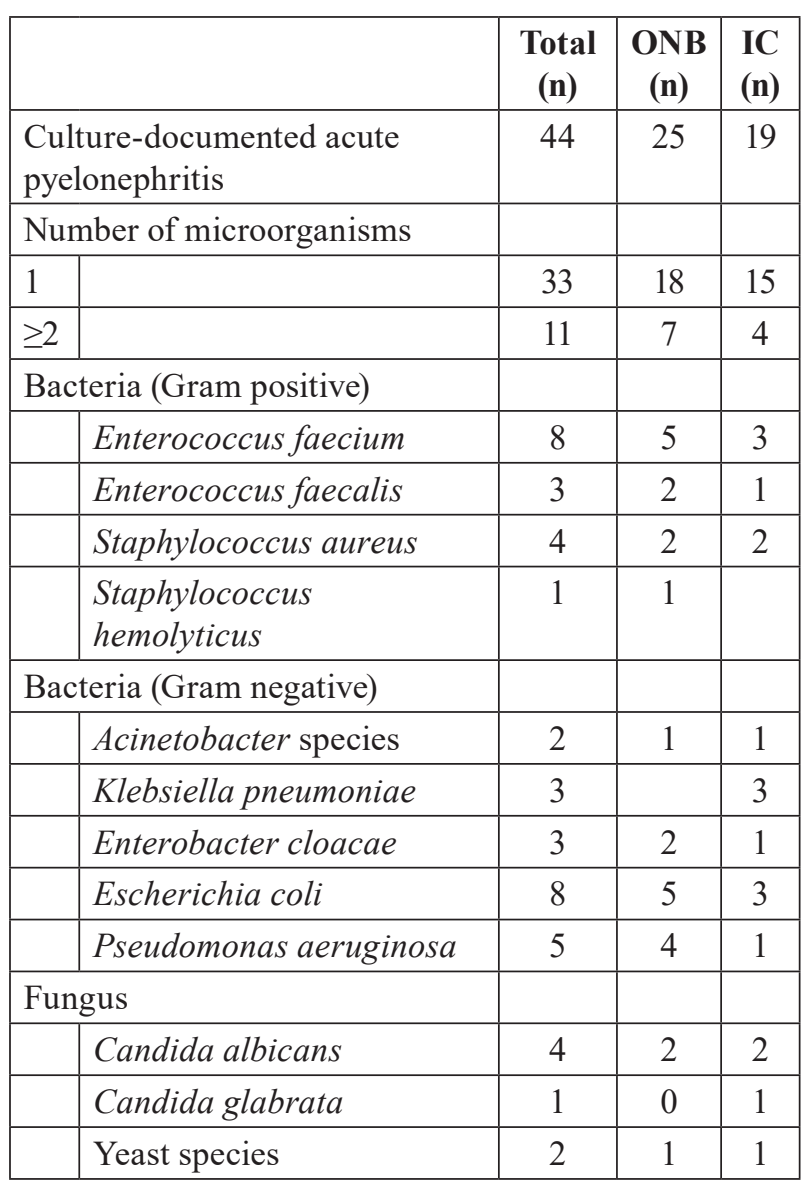

ONB, orthotopic neobladder; IC, ileal conduit. are different. In fact, several studies have included patients with asymptomatic bacteriuria. Therefore, only febrile UTI was analyzed in this study to reduce selection bias. In our study, 49 (29.9\%) patients had febrile UTI, including 24 (14.6\%) patients who had it within 3 months after the surgery.

In a nationwide population study on complications following radical cystectomy in Sweden, patients with ONB have higher risk of UTI (OR: 1.21) than those with IC. ${ }^{16}$ A single center series of 209 patients following robotic radical cystectomy with urinary diversion has also reported an increased complication risk of UTI among patients undergoing ONB. ${ }^{6}$ In this study, risk factors of symptomatic UTI included the receiving of continent urinary diversion (OR: 2.563; 95\% CI: 1.630-2.948; $p=$ $0.031)$ and the presence of diabetes (OR: $4.52 ; 95 \%$ CI: $2.862-5.571 ; p=0.002$ ).

Mechanisms underlying these results are multifactorial. Some studies have examined infections after ONB diversion and suggested the following possible mechanisms..$^{15,17,18}$ First, bacteria can colonize the bowel epithelium more easily than the urothelium and then use intestine for urinary diversion, thus increasing UTI. Second, incomplete emptying of the bladder might promote infection. Some studies have shown signi cant correlation 
between post-void residual urine volume and UTI. In addition, serial urodynamic examinations in patients with neobladders presented that a significant increase in maximal ow rate and bladder capacity concomitant to a decrease in residual urine volume were documented over a 6-month interval from surgery, possibly contributing to the decrease in UTI events. ${ }^{19}$ Third, excessive mucus production by the bowel epithelium accompanied by an established infection can prevent effective clearance of microorganisms. However, after 3-6 months after surgery, atrophic changes in the intestinal mucosa lining the neobladder occur, and consequently, the neobladder is less susceptible to bacterial colonization and infection. ${ }^{20,21}$ Finally, prolonged urinary catheterization is related to UTI. For these mechanisms, it can be suggested that UTI rate in patients with ONB decreases overtime especially after 3 months postoperatively.

UTIs in diabetic patients are common. These infections have more complex clinical course. ${ }^{22,23}$ Increased prevalence of bacteriuria in diabetic patients might be due to differences in host responses between diabetic and nondiabetic patients or difference in the infectious bacterium itself. ${ }^{22,23}$ Host-related mechanisms include the presence of urine glucose, functional change of leukocyte, and increased adhesion to urinary tract epithelium..$^{22,23}$ Some studies have reported increase of Klebsiella but decrease of E. coli in patients with diabetes. ${ }^{14,24,25}$ However, recent studies have found no signi cant difference of bacterial species in diabetic patients, ${ }^{26,27}$ while results of other studies are different. ${ }^{28}$ Our results showed that the prevalence of symptomatic UTI was increased in diabetic patients. However, such increase was not due to difference in bacterial species.

Regarding identities of microorganisms following radical cystectomy, some studies have demonstrated a predominance of Gram-negative bacteria (65-91\%). ${ }^{23,29-31}$ In patients with neobladder-related UTIs, the most common implicated microorganism is E. coli which is responsible for $59 \%$ of monobacterial infections. ${ }^{23}$ However, percentages of various bacteria were not the same in our study. Our results showed that Gram-negative bacteria were present in $47.7 \%$ of cultures. Enterococcus species, E. coli, Pseudomonas aeruginosa, Candida species, and Enterobacter species were common pathogens in this study. This could be due to different perioperative antibiotic uses, epidemiologic reasons, and different clinical characteristics of patients who previously received multiple course of antibiotic therapy and surgical procedures such as cystoscopic biopsy and transurethral resection of bladder tumor.

A positive urine culture is a common nding in patients with urinary diversion. However, this does not mean symptomatic infection. ${ }^{32}$ In general, treating an asymptomatic bacteriuria is not advocated for these patients. However, controversy exists regarding appropriate treatment of asymptomatic bacteriuria in urinary diversion patients. In a recent study, $78 \%$ of patients with ONB have pyuria and nitrates based on urinalysis. ${ }^{33}$ In the subset of patients with ONB who had evidence of UTI on urinalysis, 50\% of them developed symptomatic infection. ${ }^{33}$ Studer et al..$^{34}$ have recommended antibiotics for patients with ONB and positive urine culture result. Clearly, UTI with symptoms such as high fever, chilling, nausea, vomiting, and ank pain must be treated quickly with appropriate antibiotics. Moreover, if recurrent symptomatic UTI occurs, evaluation of ureteral obstruction or $r \quad \mathrm{ux}$ is necessary using $\mathrm{u}$ oroscopy of the reservoir or conduit to determine whether there is poor emptying of the ONB and identify stricture or obstructed areas. ${ }^{35}$ Early diagnosis and prompt drainage are required to prevent consequent renal functional loss with aggravated infectious complications.

This study has some limitations. First, it was an uncontrolled and retrospective study. Among the groups of patients, there were differences in baseline characteristics such as age, sex, and pathologic stage. This might have affected our results. However, in the selection of surgical method, there are several considerations including age, renal function, cancer stage, patient's socioeconomic status, comorbidities, and 
preference. In general, younger and healthy patients underwent $\mathrm{ONB}$ rather than IC; therefore, there were differences in characteristics between ONB and IC patients. Second, the number of patients in this study was relatively small. However, there were no signi cant differences in culture results among the groups. Therefore, comparative analysis of subgroup and culture proven microorganism is limited. Our results were different from the results of some previous studies. ${ }^{23,29-31}$ Finally, we did not analyze preoperative and postoperative changes in voiding and renal function. Ureteral stricture, obstruction, estimated glomerular ltration rate (eGFR), and voiding function are known to be associated with acute pyelonephritis. It was very hard to evaluate eGFR of the total cohort within the follow-up periods, because each patient had different health condition. Therefore, it is needed to determine long-term changes of the parameters in the future.

\section{CONCLUSION}

The results of this study revealed that at 3 months after radical cystectomy for bladder cancer, diabetes and ONB diversion signi cantly increased the risk of febrile UTI. However, our study has many limitations, including its retrospective nature, few patients with positive cultures, and different baseline characteristics among groups. Therefore, further research is needed.

\section{CONFLICTS OF INTEREST}

The authors declare no con icts of interest.

\section{FUNDING}

This research received no external funding.

\section{REFERENCES}

Revisiting the initial experience, and results in 104 patients. BJU Int 2009;103:680-3. https://doi. org/10.1111/j.1464-410X.2008.08204.x

2. Cookson MS, Chang SS, Wells $\mathrm{N}$, et al. Complications of radical cystectomy for nonmuscle invasive disease: Comparison with muscle invasive disease. J Urol 2003;169:101-4. https://doi. org/10.1016/S0022-5347(05)64045-1

3. Hautmann RE. Urinary diversion: Ileal conduit to neobladder. J Urol 2003;169:834-42. https://doi. org/10.1097/01.ju.0000029010.97686.eb

4. Lawrentschuk N, Colombo R, Hakenberg OW, et al. Prevention and management of complications following radical cystectomy for bladder cancer. Eur Urol 2010;57:983-1001. https://doi.org/10.1016/j. eururo.2010.02.024

5. Lowrance WT, Rumohr JA, Chang SS, et al. Contemporary open radical cystectomy: Analysis of perioperative outcomes. J Urol 2008;179:1313-8. https://doi.org/10.1016/j.juro.2007.11.084

6. Nazmy M, Yuh B, Kawachi M, et al. Early and late complications of robot-assisted radical cystectomy: A standardized analysis by urinary diversion type. J Urol 2014;191:681-7. https://doi.org/10.1016/j. juro.2013.10.022

7. Pariser JJ, Anderson BB, Pearce SM, et al. The effect of broader, directed antimicrobial prophylaxis including fungal coverage on perioperative infectious complications after radical cystectomy. Urol Oncol 2016;34:121.e9-14. https://doi.org/10.1016/j. urolonc.2015.10.007

8. Kang J, Baek SE, Kim T, et al. Impact of fat obesity on laparoscopic total mesorectal excision: More reliable indicator than body mass index. Int J Colorectal Dis 2012;27:497-505. https://doi. org/10.1007/s00384-011-1333-2

9. Edge SB, Compton CC. The American Joint Committee on Cancer: The 7th edition of the AJCC cancer staging manual and the future of TNM. Ann Surg Oncol 2010;17:1471-4. https://doi.org/10.1245/ s10434-010-0985-4

10. Ramakrishnan K, Scheid DC. Diagnosis and management of acute pyelonephritis in adults. Am Fam Physician 2005;71:933-42.

11. Lavallée LT, Schramm D, Witiuk K, et al. Perioperative morbidity associated with radical 
cystectomy in a multicenter database of community andacademichospitals.PLoSOne2014;9:e111281. https://doi.org/10.1371/journal.pone.0111281

12. Shabsigh A, Korets R, Vora KC, et al. De ning early morbidity of radical cystectomy for patients with bladder cancer using a standardized reporting methodology. Eur Urol 2009;55:164-74. https://doi. org/10.1016/j.eururo.2008.07.031

13. Mano R, Baniel J, Goldberg H, et al. Urinary tract infections in patients with orthotopic neobladder. Urol Oncol 2014;32:50.e9-14. https://doi. org/10.1016/j.urolonc.2013.07.017

14. Daneshmand S, Ahmadi H, Schuckman AK, et al. Enhanced recovery protocol after radical cystectomy for bladder cancer. J Urol 2014;192: 50-5. https://doi.org/10.1016/j.juro.2014.01.097

15. Parker WP, Toussi A, Tollefson MK, et al. Risk factors and microbial distribution of urinary tract infections following radical cystectomy. Urology 2016;94:96-101. https://doi.org/10.1016/j. urology.2016.03.049

16. van Hemelrijck M, Thorstenson A, Smith $P$, et al. Risk of in-hospital complications after radical cystectomy for urinary bladder carcinoma: Populationbased follow-up study of 7608 patients. BJU Int 2013;112:1113-20. https://doi.org/10.1111/bju.12239

17. Falagas ME, Vergidis PI. Urinary tract infections in patients with urinary diversion. Am J Kidney Dis 2005;46:1030-7. https://doi.org/10.1053/j. ajkd.2005.09.008

18. Mano R, Goldberg H, Stabholz Y, et al. Urinary tract infections after urinary diversion-different occurrence patterns in patients with ileal conduit and orthotopic neobladder. Urology 2018;116: 87-92. https://doi.org/10.1016/j.urology.2018.03.042

19. Wang D, Li LJ, Liu J, et al. Long-term urodynamic evaluation of laparoscopic radical cystectomy with orthotopic ileal neobladder for bladder cancer. Oncol Lett 2014;8:1031-4. https://doi.org/10.3892/ol.2014.2281

20. Di Tonno F, Siracusano S, Ciciliato S, et al. Morphological changes on the intestinal mucosa in orthotopic neobladder. Urol Int 2012;89:67-70. https://doi.org/10.1159/000338168

21. Senkul T, Yildirim S, Iseri C, wtal. Histopathologic changes in the mucosa of ileal orthotopic neobladder- ndings in 24 patients followed up for 5 years.
Scand J Urol Nephrol 2003;37:202-4. https://oi. org/10.1080/00365590310008046

22. Chen SL, Jackson SL, Boyko EJ. Diabetes mellitus and urinary tract infection: Epidemiology, pathogenesis and proposed studies in animal models. J Urol 2009;182:S51-6. https://doi.org/10.1016/j. juro.2009.07.090

23. Wilke T, Boettger B, Berg B, et al. Epidemiology of urinary tract infections in type 2 diabetes mellitus patients: An analysis based on a large sample of 456,586 German T2DM patients. J Diabetes Complications 2015;29:1015-23. https:/doi. org/10.1016/j.jdiacomp.2015.08.021

24. Keegan SJ, Graham C, Neal DE, et al. Characterization of Escherichia coli strains causing urinary tract infections in patients with transposed intestinal segments. J Urol 2003;169:2382-7. https:// doi.org/10.1097/01.ju.0000067445.83715.7b

25. Suriano F, Gallucci M, Flammia GP, et al. Bacteriuria in patients with an orthotopic ileal neobladder: Urinary tract infection or asymptomatic bacteriuria? BJU Int 2008;101:1576-9. https://oi. org/10.1111/j.1464-410X.2007.07366.x

26. Bonadio M, Costarelli S, Morelli G, et al. The in uence of diabetes mellitus on the spectrum of uropathogens and the antimicrobial resistance in elderly adult patients with urinary tract infection. BMC Infect Dis 2006;6:54. https://oi. org/10.1186/1471-2334-6-54

27. Papaza ropoulou A, Daniil I, Sotiropoulos A, et al. Urinary tract infection, uropathogens and antimicrobial resistance in diabetic and nondiabetic patients. Diabetes Res Clin Pract 2009;85:e12- 3. https://doi.org/10.1016/j.diabres.2009.04.020

28. Meiland R, Geerlings SE, De Neeling AJ, et al. Diabetes mellitus in itself is not a risk factor for antibiotic resistance in Escherichia coli isolated from patients with bacteriuria. Diabet Med 2004;21:10324. https://doi.org/10.1111/j.1464-5491.2004.01169.x

29. Hautmann RE, de Petriconi R, Gottfried HW, et al. The ileal neobladder: Complications and functional results in 363 patients after 11 years of followup. J Urol 1999;161:422-7. https://doi.org/10.1016/ S0022-5347(01)61909-8

30. Elmajian DA, Stein JP, Esrig D, et al. The Kock ileal neobladder: Updated experience in 295 
male patients. J Urol 1996;156:920-5. https://doi. org/10.1016/S0022-5347(01)65663-5

31. Arai Y, Taki Y, Kawase N, et al. Orthotopic ileal neobladder in male patients: Functional outcomes of 66 cases. Int J Urol 1999;6:388-92. https://doi. org/10.1046/j.1442-2042.1999.00084.x

32. Wullt B, Holst E, Steven K, et al. Microbial ora in ileal and colonic neobladders. Eur Urol 2004;45: 233-9. https://doi.org/10.1016/j.eururo.2003.09.002

33. Wood DP Jr, Bianco FJ Jr, Pontes JE, et al. Incidence and signi cance of positive urine cultures in patients with an orthotopic neobladder. J Urol 2003;169:2196-9. https://doi.org/10.1097/01. ju.0000067909.98836.91

34. Studer UE, Stenzl A, Mansson W, et al. Bladder replacement and urinary diversion. Eur Urol 2000;38:790-800. https://doi.org/10.1159/000020385

35. Ordorica RC, Masel J, Seigne J, et al. Evaluation and management of mechanical dysfunction in continent colonic urinary reservoirs. J Urol 2000;163:167984. https://doi.org/10.1016/S0022-5347(05)67519-2 